\title{
Prof. Robert S. Warren: come early, stay late and listen
}

Submitted Nov 04, 2019. Accepted for publication Nov 06, 2019.

doi: 10.21037/hbsn.2019.12.07

View this article at: http://dx.doi.org/10.21037/hbsn.2019.12.07

\section{Expert's introduction}

Robert S. Warren, MD (Figure 1) is a Professor of Surgery in the University of California School of Medicine and a specialist in gastrointestinal and liver cancer. He earned a medical degree at the University of Minnesota, where he completed a general surgery residency. After completing an oncology research fellowship at Memorial Sloan-Kettering Cancer Center in New York, Dr. Warren, joined UCSF Medical Center in 1988. A board-certified surgeon, he later became the Chief of Surgical Oncology at UCSF. Highly respected by his peers, Dr. Warren was named to the list of U.S. News "America's Top Doctors", a distinction reserved for the top $1 \%$ of physicians in the nation for a given specialty. His research focuses on the biology of colorectal cancer (CRC) and how it spreads.

Dr. Warren is involved in clinical and laboratory research. His clinical and laboratory-based research centers on the biology of gastrointestinal cancers, focusing on CRC metastasis and cholangiocarcinoma. His laboratory work investigates the contribution of growth factors to tumor angiogenesis in CRC and the molecular features that predict clinical outcome in primary and metastatic CRC. This work as expanded to the study of patient-derived threedimensional tumor organoids as models of drug sensitivity and resistance.

\section{Interview}

HBSN: Out of the various specialties of oncology, which made you decide to engage in the field of ICC, particularly?

Prof. Robert: My interest in oncology and surgery goes back to my days of being a fellow. I think the surgery itself was interesting and challenging and fun. But then, over time, as my clinical practice matured, I became interested in intrahepatic cholangiocarcinoma because I was frustrated that even after resection, patients don't do well. Some do, but many do not. So, the research I talked about today was really designed to try to understand the biology of human ICC, not mouse ICC, because of course, we try to cure humans, not mice.
HBSN: Can you give a general picture of your presentation to those who could not attend this meeting?

Prof. Robert: Yes, I will try to do that. The beginning of my talk emphasized that precision medicine in liver tumors is in its infancy. There are many studies looking at changing genetic changes in various cancers including ICC, that have revealed new targets for therapy. But most of the studies using drugs that target those mutations have not yet benefited many patients. The results of mutationtargeted clinical trials, with a few exceptions, have been disappointing. There are a number of possible explanations for this: sometimes the presumed driver mutation in fact is not the key element underlying progression; cancers can develop by-pass pathways that lead to resistance; and in many cases the drugs simply are not very good at affecting their targets. So, we decided to take a different approach and approach taken is that actual culture cancer science from the patient. We cultured many special ways to grow a three-dimensional sphere that can organize; we believe in self-growing in a way more closely reflecting the biology of the patient's tumor from which they will derive compared to other ways of culture itself.

\section{HBSN: Do you have any advances in your recent studies?}

Prof. Robert: We have three papers that we are preparing for the publication. Until they are published, I can't say much about them other than combination drug studies in these individualized patient-derived organoid studies in ICC have uncovered some previously unknown vulnerabilities in human cholangiocarcinoma

\section{HBSN: What are your aims for your future studies?}

Prof. Robert: I hope to take the work reviewing and apply it to patients, and we hope to screen a patient by studying his or her tumors growing as the three-dimensional organoids and select the best therapy based on the response of their tumors growing in the lab. We hope that findings from such personalized ex-vivo therapeutic trials will guide a clinical 


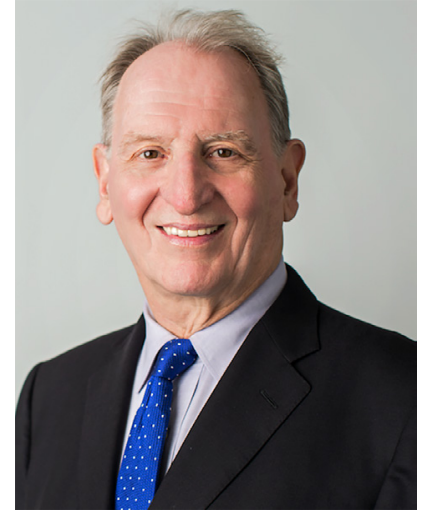

Figure 1 Prof. Robert S. Warren.

decision and inform an optimized treatment paradigm in both the adjuvant setting as well as for unresectable disease in ICC and other gastrointestinal cancers.

HBSN: As we all know, cooperation can achieve a winwin situation. So, do you have any ideas or suggestions on cooperating with Chinese medical industries?

Prof. Robert: Well, I don't have any experience with the Chinese pharmaceutical industry, but I do know that it's growing rapidly. There are many brilliant scientists as well as clinicians in China. So, it would be logical to look for areas of synergy where a collaborative interaction will be productive scientifically and clinically. So yes, I can speak for many of my colleagues at the at UCSF that there is great enthusiasm in the U.S. to explore good ideas wherever they come from.

HBSN: As one of the top doctors, do you have any words to the young who want to engage in your field?

Prof. Robert: Well, I can tell you that when I was a junior person going up to my fellowship, I asked my mentor If he had any advice. He said, "come early and stay late and listen". I think what he really meant to say is to follow your heart and your passion, don't be adverse to taking risks and you will be likely to be satisfied with a result.

\section{HBSN: When did you first hear about HBSN?}

Prof. Robert: I heard about a year ago. I had a very quick conversation with Dr. Mao, and we discussed the journal as well.

\section{HBSN: what do you think of him?}

Prof. Robert: Oh, I think he is fantastic, and he is a true leader, an inspiration for all of his trainees. He has a scientific mind great clinical acumen, and the kind of personality to make junior people watch him and wish to emulate him.

HBSN: What do you think of the journal when you first know it was launched by Chinese?

Prof. Robert: Really, only recently have I spent time reading the journal. But I can see that it is already, from the high quality of the work that was presented today, it's on its way to becoming a premier journal for hepatobiliary surgeons.

\section{HBSN: What are your expectations for HBSN?}

Prof. Robert: Well, I think it will continue to grow and really depends on the quality of papers that are submitted. It already has, as a new journal, quite high impact factor. It's not competing with Science or Nature. But it's about the same level impact as Annals of Surgery or Surgery in the United States. So, it's already coming in to its own and again I expect that it will continue to achieve more notoriety. As the quality of clinical research and scientific research submitted continues to improve, more top investigators will choose to submit their research in the journal.

\section{Acknowledgments}

I would express my heartfelt gratitude to Prof. Robert for accepting this interview. Also, thanks to $H B S N$ for giving this opportunity.

\section{Footnote}

Conflicts of Interest: The author has no conflicts of interest to declare.

(Science Editor: Ruolei Shi, HBSN, editor@thehbsn.org)

(English Language Editor: Jeremy Dean Chapnick, AME Publishing Company)

Cite this article as: Shi R. Prof. Robert S. Warren: come early, stay late and listen. Hepatobiliary Surg Nutr 2020;9(1):122-123. doi: 10.21037/hbsn.2019.12.07 